\title{
PAPER
}

\section{Implicit memory for rhythmic tone sequence: A pilot study on perceptual priming for short temporal pattern}

\author{
Yasuhiro Goto \\ School of Humanities, Hokusei Gakuen University, \\ Nishi 2-Chome 3-1 Ohyachi, Atsubetsu-ku, Sapporo, 004-8631 Japan \\ e-mail: goto@hokusei.ac.jp
}

( Received 10 May 2000, Accepted for publication 15 December 2000 )

\begin{abstract}
The aim of this study was to investigate the implicit memory for short rhythmic tone sequences from the viewpoint of cognitive psychology. The nature of memory representation for rhythmic tone sequences was researched by using priming and recognition tasks. At first, participants were asked to rate "coherence" of a rhythmic tone sequence. Then, half of the participants performed the priming task: studied and nonstudied tone sequences, both of which were of the same intensity, were presented in succession at intervals of $2 \mathrm{~s}$, and participants were asked to judge which of the two tone sequences sounded "louder." The rest of the participants performed a yes/no recognition test. The following results were obtained: 1) studied tone sequences were judged to be louder than non-studied ones in both pitch-changed and not changed conditions, 2) the priming effect decreased as the pitch changed, 3) the direction of pitch change did not influence the priming, 4) priming and recognition performance were independent of one another. These results lead to the conclusion that an implicit memory for musical rhythm exists, and pitch information is coded into the representation underlying perceptual priming of rhythmic tone sequences.
\end{abstract}

Keywords: Rhythm perception, Implicit memory, Perceptual priming, Temporal pattern,

PACS number: 43.75.Cd

\section{INTRODUCTION}

The purpose of this paper is to investigate the nature of implicit memory for rhythmic tone sequences.

In the psychological field, a great deal of recent studies about memory have been devoted to examining the relation between explicit and implicit forms of memory. Explicit memory refers to conscious or intentional recollection of previous experiences, as assessed by recall and recognition tasks; implicit memory, in contrast, refers to unconscious retrieval of previously acquired information on tests that do not require conscious recollection of a specific prior episode, such as word stem and fragment completion, lexical decision and word identification tasks. Current research has revealed dissociations between explicit and implicit memory.

Implicit memory can be confirmed by examining what is called the priming effect. Priming is a phenomenon where processing of a proceeding stimulus influences processing of a succeeding stimulus and is classified into two types: direct priming and indirect priming. Direct priming is observed when the preceding stimuli are exactly the same as the succeeding stimuli. Therefore, it is also called repetition priming or perceptual priming. In contrast, indirect priming is observed when there is a semantic relation between the preceding and succeeding stimulus. In this article, the term "priming" is used to refer to direct priming.

Research concerning implicit memory has focused almost exclusively on tests involving visual processing. Studies using verbal materials, for example, word identification (e.g., Graf and Ryan, 1990; Jacoby and Dallas, 1981), fragment and stem completion (e.g., Hayman and Tulving, 1989; Roediger and Blaxton, 1987), and lexical decision (e.g., Rueckl, 1990; Scarborough, Gerard, and Cortese, 1979) have been used as tests involving visual processing. There are also many papers about implicit memory for nonverbal objects such as picture completion (e.g., Jacoby, Baker and Brooks, 1989; Snodgrass, 1989), picture naming (e.g., Bartram, 1974; Michell and Brown, 1988), object decision (e.g., Schacter, Cooper, and Delaney, 1990), and pattern completion and identification (e.g., Musen and Treisman, 1990).

Similarly, some researches have explored implicit 
memory in the auditory domain. Several studies have demonstrated priming effects on auditory-word identification and sentence-identification tasks (e.g., Franks, Plybon, and Auble, 1982; Jackson and Morton, 1984: Schacter and Church, 1992), on an auditory stem-completion tasks (Bassili, Smith, and MacLeod, 1989; McClelland and Pring, 1991), and the like. It is true that there is relatively little research in this field, but over the past few decades a number of studies have been made on the implicit memory in the auditory domain.

For implicit memory about music, however, little attention has been focused. Only a few attempts have so far been made concerning implicit memory for music information. There are some studies about chord perception (Arao and Gyoba, 1999: Bharuha and Stoeckig, 1986, 1987; Kawaguchi and Mikumo, 1994; Tekman and Bharucha, 1992) and melody perception of alcoholic Korasakoff's syndrome patients (Johnson, Kim and Risse, 1985) and others (Peretz, Gaudreau, and Bonnel, 1998; Wilson, 1979), but as far as I know, there is no research of implicit memory for rhythmic aspects of a melody. However, it is unnatural that implicit memory for rhythmic tone sequences has not been investigated despite the fact that evidence has been found for implicit memory in various other fields. Or rather, it is reasonable to interpret that there is also implicit memory for musical rhythm perception as in other domains.

In this article, implicit memory research was extended into a previously unexplored domain: the representation and retrieval of information about rhythmic tone sequences. The most important objective of this study was to investigate whether a direct priming effect for a rhythmic tone sequence could be observed. In order to clarify the nature of implicit memory for musical rhythm, the "transformation paradigm" was used. This is a method of examining what kinds of information are important by changing all or part of the physical characteristics of stimuli between the study phase and test phase. This article concentrates especially on pitch height. That is, how pitch height information is represented in implicit memory for musical rhythm was investigated experimentally.

What sort of task could be used to assess implicit memory for rhythmic tone sequences? One promising candidate, from a number of research perspectives, is a task similar to the ones used in Mandler, Nakamura, and Zandt (1987). They used the "brightness judgment task" in order to assess implicit memory in a visual field. In their experiments, two octagons, one an old item (i.e., previously "studied" item) and the other a new item, were presented with the same brightness for very short durations. Participants were asked to judge which of the two shapes appeared brighter. The result was that participants selected the old items correctly in spite of the fact that the brightness of the two items was the same. This result was interpreted as a reflection of facilitation caused by the prior exposure; in other words, a priming effect was observed for the studied items. In this research, the task named "loudness judgment task" was used in order to assess the priming effect for rhythmic tone sequences; in the priming task listeners listened to two rhythmic tone sequences and were asked to judge which of the two tone sequences sounded "louder." If listeners selected the old item, it would be interpreted as the evidence for implicit memory for rhythmic tone sequence.

\section{METHOD}

\subsection{Design}

The experimental design consisted of one factor, a pitch shift factor: the pitch-identical condition, pitch-up condition and pitch-down condition. This factor was manipulated as a within-subject variable. Dependent measures were either performance in the priming task or in the recognition task.

\subsection{Participants}

Forty Hokkaido University undergraduates and graduates participated in the experiment.

\subsection{Apparatus}

Each tone sequence was generated on a Roland Sound Canvas SC-88 tone generator using "Cakewalk Pro Audio" software. It was controlled by a PC/AT compatible computer. The operating system was Windows 98. The tone sequences were presented through speakers ("Stage System $80 "$ made by Electro Voice Inc.).

\subsection{Materials}

The experimental materials consisted of a total of 42 tone sequences. Each of them was a rhythmic tone sequence consisting of 3-6 notes. The kinds of a note constituting the tone sequence were "eighth notes," "quarter notes," "dotted quarter notes," and/or "half notes." The real time length of the notes was 250, 500, 750 and $1,000 \mathrm{~ms}$, respectively. The total time length of each tone sequence was $2,000 \mathrm{~ms}$, and it was equivalent to " 1 measure" of 4/4 meter (see Fig. 1). They were all "equitonal" musical tone sequences, which had only note-length information, and all the other factors (e.g., pitch height, timbre, intensity) were equal. The pitch height of all these tones was the same.

These tone sequences were generated to satisfy two constrains: (a) the tone sequence consisted of more than two kinds of note values, in other words, the tone sequence did NOT consist of only one kind of note value; 

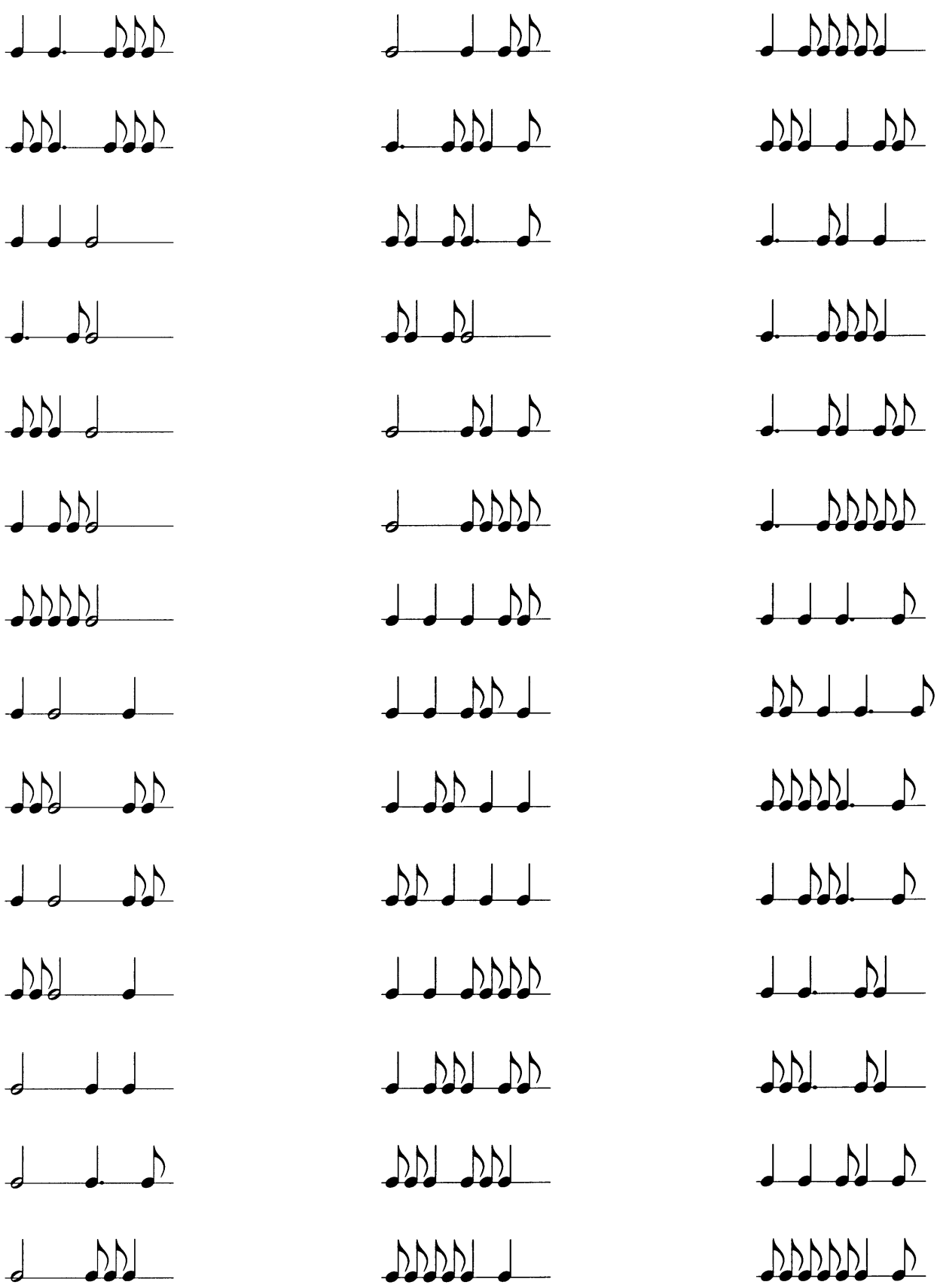

Fig. 1 Stimuli used in this experiment.

and (b) the tone sequence was metrical, not random. The reasons for these constrains were that if the tone sequence consisted of only one note value, it was too simple and not appropriate as an experimental material, and that a "nonmetrical" tone sequence was more difficult to memorize than a metrical one (cf. Povel and Essence, 1985). With regard to constraint (b), two musicians who did not participate in the present experiment rated all the candidate tone sequences in terms of how metrical each tone sequence was on a 7 -point scale $(1=$ not metrical, $7=$ metrical enough) and only tone sequences which were judged metrical were used in the present experiment.

Participants studied 21 tone sequences. The remain- ing 21 were not studied; they were included in the priming task in order to determine baseline levels of performance and in the recognition task as distracter items. The priming task and recognition task thus consisted of 42 critical items: 21 studied tone sequences and 21 nonstudied tone sequences. The presentation order of the tone sequences in both tasks was determined randomly for each participant. The sound pressure level of all tones was equal, at a comfortable listening level (about $75 \mathrm{~dB}$ SPL). The distance between each participant and the speakers was about $70 \mathrm{~cm}$. The tone sequences were played with a piano timbre. The pitch height of all notes was equal in the study phase, A4, and shifted to either E5 or B4 in the test 
phase (this is described more precisely in the procedure section).

\subsection{Procedure}

All participants were tested individually in a soundproof chamber. Each experiment was conducted under conditions of incidental encoding. Participants were told that they were participating in an experiment on the preliminary experiment of music perception, and no mention of a later memory test was made.

\subsubsection{Study phase}

In the study phase, participants were informed that rhythmic tone sequences would be presented by the speaker and their task was to rate the "coherence" of the tone sequences on a 7-point scale $(1=$ not coherent at all, $7=$ completely coherent). They were further instructed to listen to the tone sequences carefully because the tone sequences were very short, and they were told that it was important for them to make an accurate rating. The study phase then began with five practice items, followed by presentation of the 21 critical tone sequences in random order.

\subsubsection{Test phase}

Immediately after the study presentation, half of the participants were given instructions for the priming task, and the other half were given instructions for the recognition task.

The priming task was a two-forced-choice task. In each trial, 2 tone sequences (both old and new items, in random order) were presented in succession at an interval of $2.0 \mathrm{~s}$. The intensity of the two tone sequences (that is, the intensity of all notes in the tone sequences) was the same.

The pitch of one third of the old items, or 7 tone sequences in the priming task, was raised by shifting all pitches 5 scale steps upward from A4 to E5. Similarly, the other 7 tone sequences were lowered by shifting all pitches down from A4 to D4. The pitch height of the old and new items was the same in each trial. Participants were told that their task was to judge which of the tone sequences was louder. Participants were instructed to listen carefully and mark on the designated place on the sheet.

The recognition task was a surprise yes/no task. Participants were instructed to mark either "Hai" ("yes" in Japanese) on the sheet if they remembered hearing the tone sequence during the prior rating task, or "Iie" ("no" in Japanese) if they did not remember hearing the tone sequence.

The pitch of one-third of the old items, or 14 tone sequences in the recognition task, was raised by shifting all pitches upward from A4 to E5. Similarly, the other 14 tone sequences were lowered by shifting all pitches 5 scale steps down from A4 to D4. Six practice items (three new and three old) were presented before the 42 critical items. The order of presentation was randomized. As in the priming task, a period of about $1 \mathrm{~min}$ intervened between the completion of the study task and the appearance of the first critical item on the recognition test. The exact length of time to complete the recognition task varied from participant to participant, but it generally took about $10 \mathrm{~min}$.

After the completion of the test, all participants were debriefed about the nature and purpose of the experiment.

\section{RESULTS}

The results of the priming and recognition tasks are first considered separately and then followed by a contingency analysis of the relation between them.

\subsection{Priming Task}

Because the priming task was a two-forced-choice task, only the hit rate was analyzed. The hit rate was the proportion of studied items called "louder." The priming effect was defined as the difference between the hit rate and the chance level (50\%, Fig. 2).

Three important points should be noted about the results of the priming task. First, the priming effect was observed in all conditions. Participants selected the studied items correctly in the pitch-identical condition, the pitchup condition and the pitch-down condition. Second, the priming effect in the pitch-identical condition was larger than that in the pitch-up condition and the pitch-down condition. Third, the priming effect was almost the same in the pitch-up condition and the pitch-down condition. These results show that the direction of the shift of pitch did not have an influence on the magnitude of the priming effect.

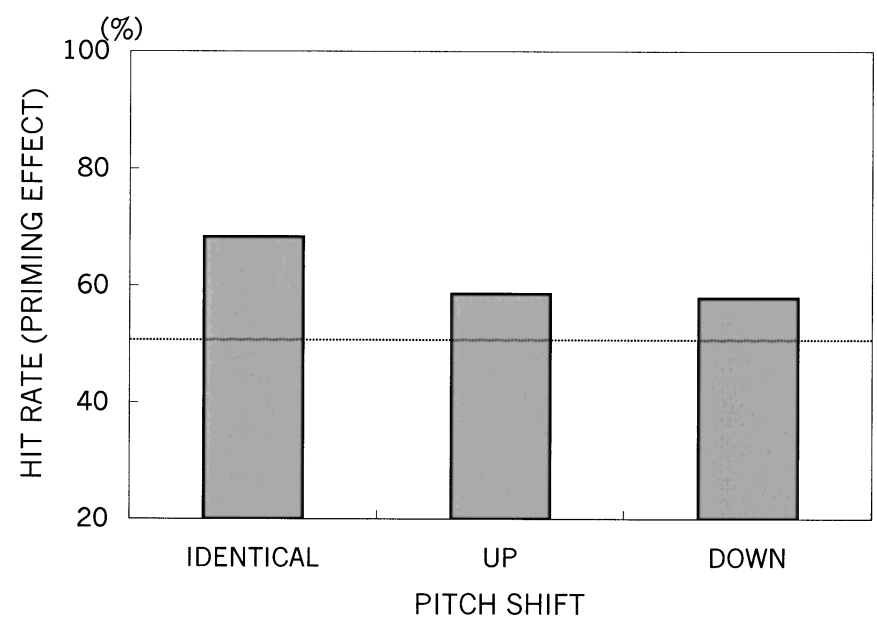

Fig. 2 The result of the priming task. The dotted line represents the chance level of $50 \%$. 
Table 1 Recognition performance in each conditions and items.

\begin{tabular}{lcccc}
\hline & \multicolumn{3}{c}{ Condition } \\
\cline { 2 - 5 } Item type & Identical & Pitch up & Pitch down & \\
\hline Studied & 0.523 & 0.498 & 0.481 & 0.501 \\
Nonstudied & 0.499 & 0.501 & 0.486 & 0.495 \\
$M$ & 0.511 & 0.500 & 0.484 & \\
\hline
\end{tabular}

Analysis of variance (ANOVA) confirmed this description of the results. A significant main effect of a pitch shift was observed $(F(2,38)=12.33, p<0.01)$. Tukey's HSD test showed significant differences between the pitch-identical condition and the pitch-up condition and between the pitch-identical condition and the pitchdown condition $(H S D=0.24, p<0.01, H S D=0.19$, $p<0.01$, respectively). No significant difference was observed between the pitch-up condition and the pitch-down condition.

A further analysis was performed in order to confirm the difference between the results of the hit rate and the chance level in each condition. In all conditions, $t$-test revealed that the result of the hit rate was significantly higher than the chance level $(t(19)=3.11, p<0.001$, $t(19)=2.96, p<0.001, t(19)=3.21, p<0.001$, respectively).

\subsection{Recognition Task}

Table 1 shows the results of the recognition performance. Two different measures of recognition were subjected to an ANOVA: the hit rate and hit rate minus false alarm rate. Since both analyses led to an identical conclusion, only the results of the hit rate analysis were reported; this simply reflects the fact that false alarm rates were relatively constant across all conditions.

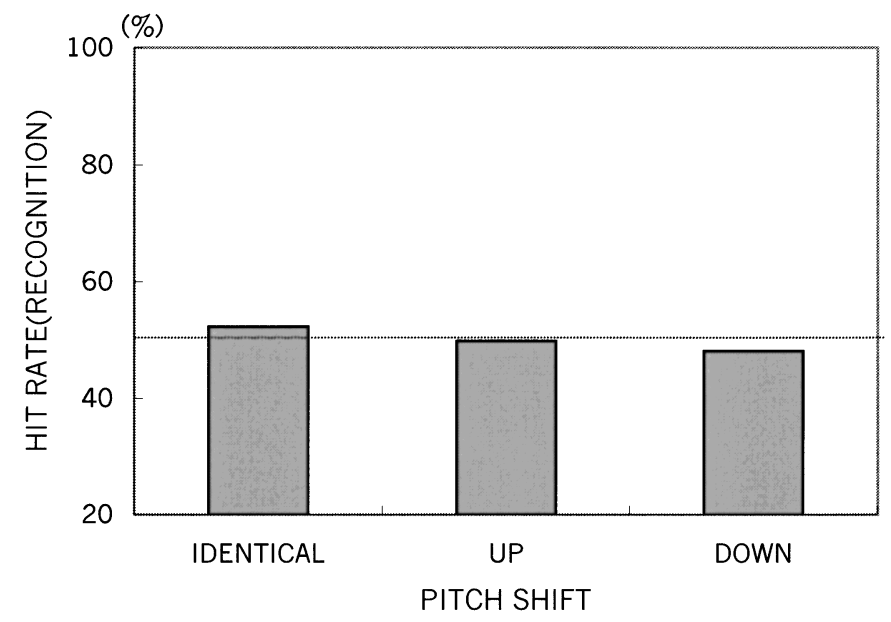

Fig. 3 The result of the recognition task. The dotted line represents the chance level of $50 \%$.
An overall ANOVA revealed no significant main effect of pitch shift. The difference between the results of the hit rate and the chance level in each condition was not significant (Fig. 3).

\subsection{Contingency Analysis of Coherence Judgment and Recognition Performance}

The purpose of the contingency analysis was to determine whether priming effects on coherence performance are dependent on, or independent of, recognition memory. In order to determine the relation between priming task and recognition task, the Yule $Q$ statistic was used. $Q$ is a measure of the strength of relation between two variables that can vary from -1 (negative association) to +1 (positive association); 0 indicates complete independence (Hayman and Tulving, 1989). For the present data, $Q=+0.082$ at the pitch identical condition, +0.099 at the pitch up condition, and +0.099 at the pitch-down condition. These values did not differ significantly from zero; significance was assessed by a chi-square test suggested by Hayman and Tulving, $\chi^{2}(1, N=20)=0.52$ at the pitch-identical condition, $\chi^{2}(1, N=20)=0.424$ at the pitch-up condition, and $\chi^{2}(1, N=20)=0.48$ at the pitch-down condition. The contingency analysis thus demonstrates stochastic independence between recognition and coherence judgment performance.

\section{DISCUSSION}

In this study the implicit memory for music was investigated experimentally.

A priming task and recognition task were performed for musical rhythm, that is to say, the rhythmic tone sequences consisted of more than one note value. The following results were obtained in the priming task: First, perceptual priming was observed not only in the pitchidentical condition but also in both the pitch-up and pitchdown conditions. The results show that information that is independent of the pitch height is encoded in the memory representation of a rhythmic tone sequence.

Secondly, the result of priming in both the pitch-up condition and the pitch-down condition was smaller than that of the priming effect obtained in the pitch-identical condition. This result indicates that information depending on the pitch height of the stimuli is encoded in the 
memory representation. These results lead to the conclusion that the memory representation of the rhythmic tone sequence encodes information both dependent and independent of the pitch height.

Thirdly, the magnitude of the priming effect was the same in both the pitch-up condition and the pitch-down condition. In other words, the direction of the pitch shift did not influence the priming effect. These results can be explained as follows; the priming effect in both the pitchup and pitch-down conditions is caused only the information independent of the pitch shift because information dependent on the pitch shift is lost by raising or lowering the pitch height, and such information is independent of the pitch shift.

In the recognition task, the results were at the chance level in all conditions. Coherent judgment performance seems not to have been influenced by the recognition performance because the results of the recognition task were statistically independent of the priming task.

For the moment let us look closely at the pitch height information in implicit memory for rhythm. The results show that such perceptual and surface feature as pitch height of rhythm is retained in the implicit memory. These results agree with those obtained by some previous works, for example, Schacter and Church (1992). They reported that the information on voice type (male or female voices) was preserved in the implicit memory but not in the explicit memory. Similarly, in this experiment, pitch height information was retained only in the implicit memory and not in the explicit memory.

A plausible account for these findings can be found in the concept of the perceptual representation system (PRS: e.g., Hyman and Tulving, 1989: Schacter, 1990, 1992; Tulving and Schacter, 1990). PRS is composed of a number of subsystems that process information about presemantic features like form, structure, and so on, but not the meaning and associative properties of words, objects, and other types of stimuli. Although the PRS frameworks have focused on visually based subsystems, neuropsychological research suggests the existence of a form versus semantic dissociation within the auditory domain analogous to those observed within the visual domain (Ellis, 1982;, Ellis and Young, 1988). The results of this experiment can also be explained on similar lines. Accordingly, surface and perceptual information such as the pitch height of rhythm would be processed at the presemantic level of the auditory memory system.

This study has yielded experimental facts about implicit memory for rhythm. As stated at the outset, almost all of the research on implicit memory for music has focused on pitch height aspects, but not on rhythmic aspects. Why has much research on implicit mem- ory for music treated pitch height aspects? One reason is that it may be relatively easy to design experiments by ignoring rhythm. For example, studies of chord priming showed evidence of implicit memory by taking advantage of the "simultaneity" of notes and did not consider rhythm (e.g., Arao and Gyoba, 1999: Bharuha and Stoeckig, 1986, 1987; Kawaguchi and Mikumo, 1994; Tekman and Bharucha, 1992). In other words, they did not take into account the time aspect, that is, the passage of time. It seems reasonable to suppose that such an experimental paradigm is analogous to memory research in visual field, where other factors are considered to be more important and the time aspect is, in fact, often ignored.

Comparing with those studies, it is difficult to investigate the nature of implicit memory for musical rhythm because "rhythm" is the time aspect of music itself and it is impossible to ignore that aspect in studying rhythm. This study, however, overcame such a difficulty by using the "loudness judgment task" method, and it succeeded in clarifying the nature of the implicit memory for musical rhythm to some extent. It is true that loudness judgment task may not be a general method for examining implicit memory, but it can be a useful method for studying implicit memory for rhythm.

This research examined implicit memory for musical rhythm, and the nature of memory representation for rhythmic tone sequences was clarified focusing especially on the pitch height of notes. Needless to say, we cannot say that the whole nature of memory representation for rhythmic tone sequence was clarified by only the experiment described above. There is room for further investigation; for example, whether the degree of pitch shift has an influence on the magnitude of the priming effect, whether other information about notes, such as timbre, intensity and so on, can be represented in the memory representation, and whether a non-metrical rhythmic tone sequence has a similar priming effect. These remain to be proved in future investigations.

\section{REFERENCES}

Arao, H. and Gyoba, J. (1999). "Disruptive effects in chord priming", Music Percept. 17, 241-245.

Bartram, D. J. (1974). "The role of visual and semantic codes in object naming", Cognit. Psychol. 6, 325-356.

Bassili, J. N., Smith, M. C. and MacLeod, C. M. (1989). "Auditory and visual word-stem completion: Separating data-driven and conceptually driven process", Q. J. Exp. Psychol. 41A, 439-453.

Bharucha, J. J. and Stoeckig, K. (1986). "Reaction time and musical expectancy: Priming of chords", J. Exp. Psychol. Hum. Percept. Perform. 12, 403-410.

Bharucha, J. J. and Stoeckig, K. (1987). "Priming of chords: Spreading activation or overlapping frequency specta?", Percept. Psychophys. 41, 519-524.

Ellis, A. (1982). "Modality-specific repetition priming of auditory word recognition", Curr. Psychol. Res. 2, 123-128. 
Ellis, A. and Young, A. (1988). Human Cognitive Neuropsychology (Erlbaum, Hillsdale, NJ).

Franks, J. J., Plybon, C. J. and Auble, P. M. (1982). "Units of episodic memory in perceptual recognition", Mem. Cognit. 10, 62-28.

Graf, P. and Ryan, L. (1990). "Transfer-appropriate processing for implicit and explicit memory”, J. Exp. Psychol. Learn. Mem. Cogn. 16, 978-992.

Hyman, C. A. G. and Tulving, E. (1989). "Contingent dissociation between recognition and fragment completion: The method of triangulation", J. Exp. Psychol. Learn. Mem. Cogn. 15, 228-240.

Jackson, A. and Morton, J. (1984). "Facilitation of auditory word recognition", Mem. Cognit. 12, 568-574.

Jacoby, L. L., Baker, J. G. and Brooks, L. R. (1989). "Episodic effects on picture identification: Implications for theories of concept learning and theories of memory", J. Exp. Psychol. Learn. Mem. Cogn. 15, 275-281.

Jacoby, L. L. and Dallas, M. (1981). "On the relationship between autobiographical memory and perceptual learning”, J. Exp. Psychol. Gen. 110, 306-340.

Johnson, M. K., Kim, J. K. and Risse, G. (1985). "Do alcoholic Korasakoff's syndrome patiendts acquire affective reactions?", $J$. Exp. Psychol. Learn. Mem. Cogn. 11, 22-36.

Kawaguchi, J. and Mikumo, M. (1994). "Implicit memory for music information: Priming effect on majar-minor decision task for chord", Abstr. 3rd Practical Aspects of Memory Conf., Mayland, U.S.A., p. 107.

MaClelland, A. G. R. and Pring, L. (1991). "An investigation of cross-modality effects in implicit and explicit memory", Q. J. Exp. Psychol. 43A, 19-33.

Mandler, G., Nakamura, Y. and Zandt, B. J. S. V. (1987). "Nonspecific effects of exposure on stimuli that cannot be recognized", $J$. Exp. Psychol. Learn. Mem. Cogn. 13, 646-648.

Mitchell, D. G. and Brown, A. S. (1988). "Persistent repetition priming in picture naming and its dissociation from recognition memory”, J. Exp. Psychol. Learn. Mem. Cogn. 14, 213-222.

Musen, G. and Treisman, A (1990). "Implicit and explicit memory for visual patterns", J. Exp. Psychol. Learn. Mem. Cogn. 16, 127137.

Peretz, I., Gaudreau, D. and Bonnel, A. M. (1998). "Exposure effects on music preference and recognition”, Mem. Cognit. 26. 884-902.
Povel, D. J. and Essens, P. (1985). "Perception of temporal patterns", Music Percept. 2, 411-440.

Roediger, H. L. III and Blaxton, T. A. (1987). "Effects of varying modality, surface features, and retention interval on priming in word-fragment completion", Mem. Cogn. 15, 379-388.

Rueckl, J. G. (1990). "Similarity effects in word and pseudoword repetition priming”, J. Exp. Psychol. Learn. Mem. Cogn. 16, 374391.

Scarborough, D. L., Gerard, L. and Cortese, C. (1979). "Accessing lexical memory: the transfer of word repetition effects across task and modality", Mem. Cognit. 7, 3-12.

Schacter, D. L. (1990). "Perceptual representation systems and implicit memory: Toward a resolution of the multiple memory systems debate", in Development and Neural Bases of Higher Cortical Functions, A. Diamond, Ed.: Ann. N.Y. Acad. Sci. 608, 543571.

Schacter, D. L. (1992). "Understanding implicit memory: A cognitive neuroscience approach", Am. Psychol. 47, 559-569.

Schacter, D. L. and Church, B. A. (1992). "Auditory priming: implicit and explicit memory for words and voices", J. Exp. Psychol. Learn. Mem. Cogn. 18, 915-930.

Schacter, D. L., Cooper, L. A. and Delaney, S. M. (1990). "Implicit memory for unfamiliar objects depends on access to structural descriptions", J. Exp. Psychol. Gen. 119, 5-24.

Snodgrass, J. G. (1989). "Sources of learning in the picture fragment completion task", in Implicit Memory: Theoretical Issues, S. Lewandowsky, J. C. Dunn and K. Kirsner, Eds. (Erlbaum, Hillsdale, NJ), pp. 259-282.

Tekman, H. G. and Bharucha, J. J. (1992). "Time course of chords priming", Percept. Psychophys. 51, 33-39.

Tuving, E. and Schacter, D. L. (1990). "Priming and human memory systems", Science 247, 301-306.

Wilson, W. R. (1979). "Feeling more than we can know: Exposure effects without learning", J. Pers. Soc. Psychol. 37, 811-821.

Yasuhiro Goto was born in 1968. He received the B.E. degree in Psychology from University of Tokyo in 1993, and M.E and Ph.D degrees in Behavioral Science from Hokkaido University. He is a member of the JPA (Japanese Psychological Association), JCSS (Japanese Cognitive Science Society), JSMPC (Japanese Society for Music Perception and Cognition), and ASJ. 